“ C 2019 IEEE. Personal use of this material is permitted. Permission from IEEE must be obtained for all other uses, in any current or future media, including

reprinting/republishing this material for advertising or promotional purposes, creating new collective works, for resale or redistribution to servers or lists, or reuse of any copyrighted component of this work in other works." 


\title{
Circular-Polarized Substrate-Integrated-Waveguide Leaky-Wave Antenna with Wide-Angle and Consistent-Gain Continuous Beam Scanning
}

\author{
Shu-Lin Chen, Student Member, IEEE, Debabrata K. Karmokar, Member, IEEE, Zheng Li, Member, IEEE, \\ Pei-Yuan Qin, Member, IEEE, Richard W. Ziolkowski, Fellow, IEEE, and Y. Jay Guo, Fellow, IEEE
}

\begin{abstract}
Circularly-polarized (CP) antennas are highly desired for future wireless communications. To advance the development of CP substrate-integrated-waveguide (SIW) leakywave antennas (LWAs) with the intent to meet this demand, a novel benzene-ring-shaped slot-loaded LWA with partially reflecting wall (PRW) vias is investigated and verified to realize wide-angle continuous beam scanning with consistent gain. The dispersion features of slot-loaded SIW LWAs with PRW vias are theoretically explored using an equivalent circuit model. The CP radiation feature is investigated numerically utilizing the $\mathrm{E}$ - and $\mathrm{H}$-field distributions of an initial design and its equivalent magnetic currents. The results of these studies are used to demonstrate that improved $\mathrm{CP}$ performance over a wideangle scan range can be attained with a change from a standard slot shape to a benzene-ring-shaped slot. The resulting benzenering-shaped slot-loaded CP SIW LWA was optimized, fabricated, and measured. The measured results verify that a $\mathrm{CP}$ beam was continuously scanned through a wide angle from backward to forward directions with a consistent gain. The prototype exhibits a continuous $97.1^{\circ} \mathrm{CP}$ beam scan with a gain variation between 8 and 11.3 dBic when the source frequency is swept from 9.35-11.75 GHz.
\end{abstract}

Index Terms-Circular polarization (CP), consistent gain, continuous beam scanning, leaky-wave antenna (LWA), substrate integrated waveguide (SIW).

\section{INTRODUCTION}

$\mathbf{L}$ EAKY-wave antennas (LWAs) are highly promising candidates for future wireless communications and sensing systems based on emerging platforms such as drones and small satellites, where low-cost and low-profile antennas are required. Generally, they radiate power as a traveling wave propagates across "open" regions along its aperture. Their main beam directions can be steered by varying their source frequencies [1], [2]. Correspondingly, LWAs can bring significant benefits including a simple feed network, wide-angle beam scanning, a low profile and cost effectiveness [3]-[7]. However, for applications to radar and satellite communication systems, it is also preferred to radiate a circularly polarized

Manuscript received XXX. This work was supported by the Australian Research Council (ARC) Discovery Project (DP) 160102219 grant, the Discovery Early Career Researcher Award (DECRA) DE170101203 grant, and the National Natural Science Foundation of China under Grant 61771038. (Corresponding author: Shu-Lin Chen)

The authors are with the Global Big Data Technologies Centre (GBDTC), University of Technology Sydney (UTS), Ultimo, NSW 2007, Australia. (Email: Shulin.Chen@uts.edu.au).

$\mathrm{Z}$. Li is also with the Institute of Lightwave Technology, Beijing Jiaotong University, Beijing 100044, China. (E-mail: lizheng@bjtu.edu.cn)
(CP) wave rather than a linearly polarized (LP) one, i.e., the CP LWAs are highly desired to avoid polarization mismatch and to suppress multi-path interference during beam scanning [8]-[10].

Some challenges exist in achieving frequency-dependent, continuous beam scanning from a CP LWA. First, it is known that for the LWAs working at frequency $f$, the main beam angle $\theta(f)$ in its leaky region is determined with the relation:

$$
\theta(f)=\sin ^{-1}\left[\beta(f) / k_{0}(f)\right]
$$

where $\beta(f)$ is the phase constant of the leaky wave mode in the guiding structure, and $k_{0}(f)$ is the corresponding propagation constant in air. In order for it to scan the main beam from the backward to the forward direction, a CP LWA must facilitate both positive and negative phase constants. Second, the radiated fields must have two perpendicular components with the same amplitude and a $90^{\circ}$ phase difference to be $\mathrm{CP}$ waves. This feature must be maintained within the main beam direction as the frequency changes. Moreover, it is known that most periodic LWAs suffer from the open stopband (OSB) problem, i.e., when their beam points at the broadside direction, their realized gain drops significantly [11]-[13]. Furthermore, there are linkages between suppressing the OSB and the consequent $\mathrm{CP}$ performance [14]. On the other hand, even if one does suppress the OSB, it remains a challenge to maintain a consistent gain performance over the entire beam scanning range. Thus, a high performance CP LWA must also have solutions for all of these issues.

A planar-modulated surface-based CP LWA has been reported that suppresses the OSB. However, it is unable to scan its main beam [15]. There are some other interesting approaches that also accomplish continuous $\mathrm{CP}$ beam scanning through broadside. One technique is to introduce composite right/left-handed (CRLH) unit cells that satisfy their balanced condition. The OSB is suppressed, and the main beam can be scanned in its fundamental mode [16]-[18]. Moreover, by introducing a substrate-integrated-waveguide (SIW) or a microstrip line structure into these CRLH-based LWAs, continuous backward to forward $\mathrm{CP}$ beam scanning can be obtained [19]-[22]. Using a half-mode (HM) SIW etched with rampshaped slots, a wide-angle continuous backward-to-forward beam scanning was obtained in [21]. Another technique is to use axial-asymmetrical microstrip lines [14], [20]. These series-fed microstrip LWAs introduce an asymmetry in the 
transversal axis to radiate and scan the CP beam as the source frequency is varied. Nevertheless, it is still very challenging to simultaneously achieve good axial ratio (AR) values and a suppressed OSB over a wide angle scan range. A third technique is to employ spoof surface plasmon polaritons (SSPPs) [23], [24]. Circular patches located above a slowwave SSPP line were reported in [23] to generate the CP wave, and the main beam was continuously scanned from $-32^{\circ}$ to $+34^{\circ}$ with gain variations between $12.8-14.2 \mathrm{dBic}$. Yet another popular approach is to use a periodic slot-loaded aperture in a SIW structure. These SIW LWAs have been widely investigated and used due to their easy integration and low loss [25], [26]. An H-shaped slot-loaded SIW reported in [27] realized beam scanning in the forward quadrant from $20^{\circ}$ to $50^{\circ}$ when the source frequency varied between 10.6 and 11.5 GHz. A slot-loaded periodic SIW CP LWA was reported in [28] that successfully realized continuous beam scanning from $-40^{\circ}$ to $+25^{\circ}$ through broadside by manipulating the etched slots on the SIW. Again, the realized gain had a large variation between 4.2 to $11 \mathrm{dBic}$ within the scanning range. As all of these examples illustrate, it is a very challenging problem to achieve a wide-angle continuous CP beam scan with consistent gain performance.

In this paper, a novel CP LWA based on a periodic benzenering-shaped slot-loaded SIW with partially reflecting wall (PRW) vias is introduced. Analysis and simulation studies demonstrate that it achieves a wide-angle, consistent-gain, continuous beam scanning from backward to forward directions through broadside. A prototype of the optimized design was fabricated and tested. The measured results confirm that it can continuously scan the main beam through a wide angle, ranging from $-42.8^{\circ}$ to $+54.3^{\circ}$, with only a $3.3 \mathrm{~dB}$ gain variation. The main contributions of this work include the incorporation of both PRW vias and slot elements to substantially enhance the radiation performance and a thorough investigation of the $\mathrm{CP}$ beam radiation performance with both equivalent circuits and equivalent radiating sources. Finally, when it is compared to most of the reported CP LWAs, the developed benzene-ringshaped slot-loaded CP SIW LWA with PRW vias exhibits a better beam-scanning range and gain variation performance.

\section{Unit Cell Requirements to AChieve Continuous BEAM SCANNING}

Initially, a non-slotted SIW unit cell with partially reflecting wall (PRW) vias was investigated. It is labelled as Unit A in Fig. 1. It is designed on a two-layer copper-clad substrate. The substrate is chosen as F4BM-2 produced by Wangling Company, and the permittivity $\left(\varepsilon_{r}\right)$, loss tangent $(\tan \delta)$, width $\left(w_{s u b}\right)$ and thickness are 3.02, 0.001, $30 \mathrm{~mm}$ and $1 \mathrm{~mm}$, respectively. A main patch with a width of $w_{p}$ is printed on top of the substrate, and a solid ground plane is placed at the bottom. Two rows of shorting vias are used for connecting the patch to the ground plane. The shorting vias in the upper row have a diameter $d_{1}$ and a center-to-center distance $S$. They act as a electric wall of the SIW LWA. The shorting vias in the lower row have a diameter $d_{2}$ and are separated by a larger distance $P$ and serve as the PRW. The length of unit cell is also $P$.

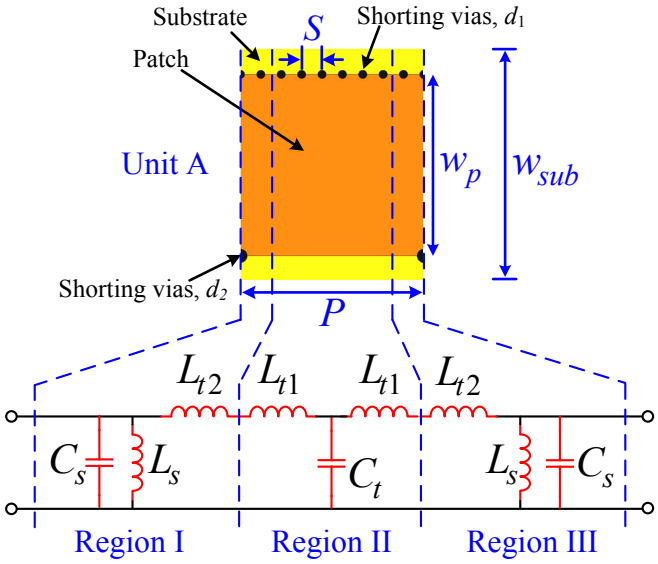

Fig. 1. Top view of the unit cell, Unit A, and its corresponding equivalent circuit.

The analysis of the unit cell is conducted using transmission line theory [29]. The corresponding Unit A equivalent circuit is depicted as shown in Fig. 1. For clearer explanations, the entire unit cell is divided into three regions, marked as Regions I, II and III. The series inductances $L_{t 1}$ and $L_{t 2}$ are introduced by the indicated regions of the main patch, and the shunt capacitances $C_{t}$ and $C_{S}$ are caused by the the capacitive effects between the corresponding regions and the ground plane. The shunt inductance $L_{s}$ is introduced by the shorting vias. Due to the placement of the series inductances $L_{t 1}$ and $L_{t 2}$, a total inductance of $L_{t}$ is used for simplicity in the analysis.

The transmission (ABCD) matrix of Unit A is then calculated as:

$$
\begin{aligned}
{\left[\begin{array}{ll}
A & B \\
C & D
\end{array}\right] } & =\left[\begin{array}{cc}
1 & 0 \\
1 /\left(j \omega L_{s}\right) & 1
\end{array}\right] \times\left[\begin{array}{cc}
1 & 0 \\
j \omega C_{s} & 1
\end{array}\right] \\
& \times\left[\begin{array}{cc}
1 & j \omega L_{t} \\
0 & 1
\end{array}\right] \times\left[\begin{array}{cc}
1 & 0 \\
j \omega C_{t} & 1
\end{array}\right] \times\left[\begin{array}{cc}
1 & j \omega L_{t} \\
0 & 1
\end{array}\right] \\
& \times\left[\begin{array}{cc}
1 & 0 \\
j \omega C_{s} & 1
\end{array}\right] \times\left[\begin{array}{cc}
1 & 0 \\
1 /\left(j \omega L_{s}\right) & 1
\end{array}\right]
\end{aligned}
$$

where, $\omega$ is the angular frequency $\omega=2 \pi f$, and $L_{t}=L_{t 1}+$ $L_{t 2}$. The dispersion characteristic is expressed in terms of the transmission line parameters as [30]:

$$
\beta=\frac{1}{P} \cos ^{-1}\left(\frac{A+D}{2}\right)
$$

By solving (2) and (3), the wave number $\beta$ can be represented as:

$$
\begin{aligned}
\beta= & \frac{1}{P} \cos ^{-1}\left[-C_{t} L_{t}^{2} C_{s} \omega^{4}-\left(2 L_{t} C_{s}+L_{t} C_{t}\right.\right. \\
& \left.\left.+\frac{C_{t} L_{t}^{2}}{L_{s}}\right) \omega^{2}+2 L_{t} L_{s}+1\right]
\end{aligned}
$$

To obtain the circuit parameter values, one can extract the approximate total patch inductance $L$ and total capacitance $C$ of Unit A using the following equations [31]:

$$
L \approx \mu_{r} \mu_{0} P h / w_{p}, \quad \text { and } \quad C \approx \varepsilon_{r} \varepsilon_{0} w_{p} P / h
$$

where $\mu_{r}$ is the relative permeability of the substrate, $\mu_{0}$ is the permeability of free space, $\varepsilon_{r}$ is the relative permittivity 


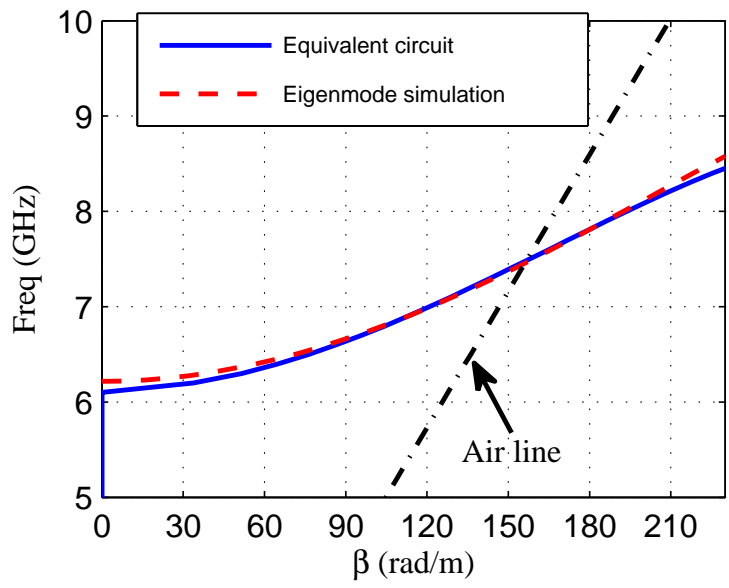

Fig. 2. Dispersion diagrams of the Unit A model obtained with equivalent circuit and eigenmode simulations.

of the substrate, $\varepsilon_{0}$ is the permittivity of free space, and $h$ is the thickness of the substrate. The values (in millimeters) of the Unit A parameters are: $w_{p}=10.7, P=10.8, S=1.2$, $d_{1}=0.5, d_{2}=1$. Substituting these parameter values into (5), the resultant inductance $L \approx 1 \mathrm{nH}$ and capacitance $C \approx 3.3$ $\mathrm{pF}$ are obtained. As $L=2 L_{t}$ and $C=2 C_{s}+C_{t}$, the values for $L_{t}, C_{s}$ and $C_{t}$ become: $C_{s}=1 \mathrm{pF}, L_{t}=0.5 \mathrm{nH}, C_{t}=1.3$ pF. Furthermore, the inductance $L_{p}$ of the PRW vias can be approximately calculated as [32]:

$$
L_{p} \approx 0.2 h\left(\ln \frac{4 h}{d_{2}}+1\right) \times 10^{-6}
$$

Then by substituting the values of $h$ and $d_{2}$ into it and considering the parallel inductive effects of the shorting vias in the upper row, the inductance $L_{s}$ of the shorting vias is approximately obtained as: $L_{s} \approx 0.3 \mathrm{nH}$.

Applying these calculated circuit parameter values to (4), one can calculate its dispersion feature. The results are labeled as Equivalent circuit in Fig. 2. As a comparison, Unit A is also simulated using the eigenmode solver in the full-wave finite element (FEM) simulator ANSYS High Frequency Structure Simulator (HFSS). The resultant dispersion diagram is labeled as Eigenmode simulation in Fig. 2. It is seen that the dispersion diagram obtained from the equivalent circuit reasonably agrees with the one from the eigenmode simulation. Referring to Fig. 2, it is known that the left region of the air line is the leaky wave region, i.e., $\beta \leq k_{0}$. Thus, the LWAs employing Unit A can only radiate forward beams at its fundamental $(n=0)$ mode.

In our previous paper [33], a slot-loaded SIW LWA with PRW vias was reported that produced a continuously scanned LP beam. To achieve a continuous backward-to-forward scanned CP beam, a new unit cell (referred as Unit B) is developed; it is depicted in Fig. 3. Unit B has an I-shaped slot centered on the main patch. As indicated, the dimensions of the slot are $w_{s}, l_{h}$, and $l_{v}$. Based on the Unit $\mathrm{A}$ analysis, the Unit B equivalent circuit is given in Fig. 3. The equivalent circuits corresponding to Regions I and III are the same as those of Unit A. In contrast, it is different in Region II. The shunt

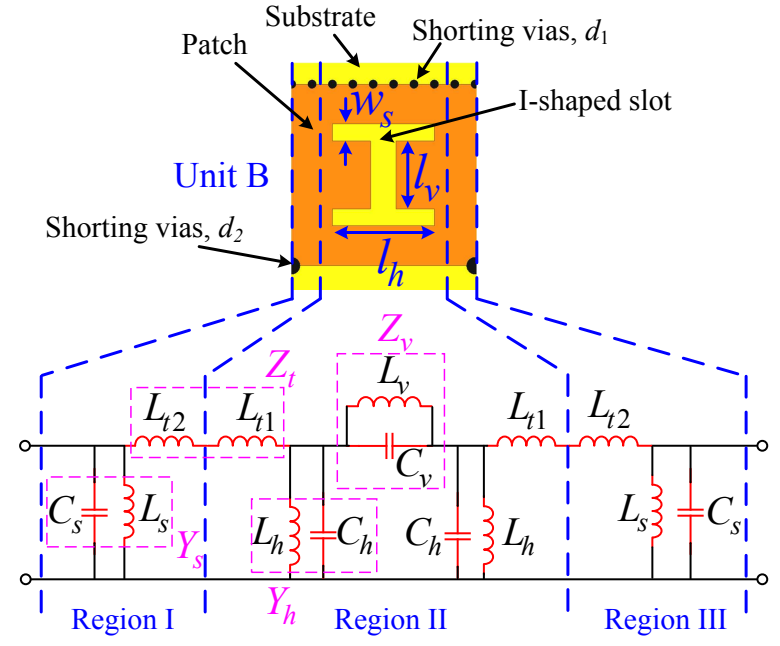

Fig. 3. Unit B model and its corresponding equivalent circuit.

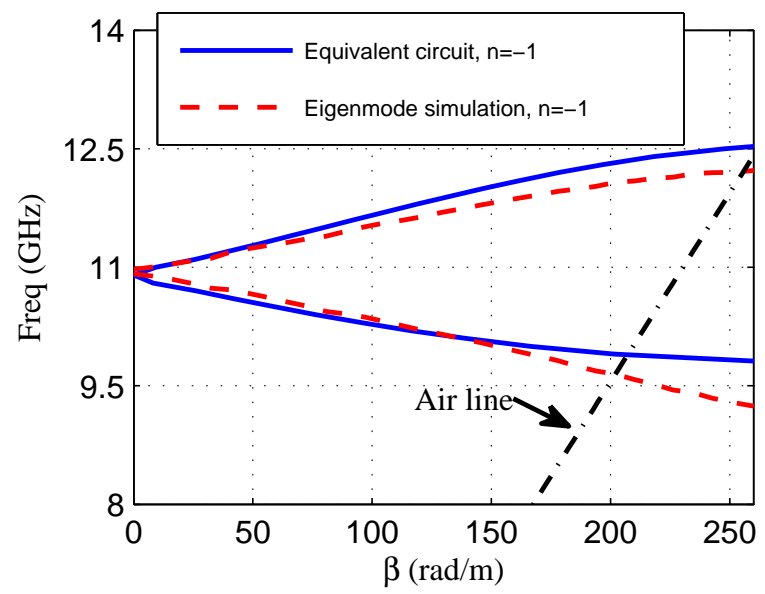

Fig. 4. Unit B dispersion diagrams obtained from its equivalent circuit and eigenmode simulation.

inductance $L_{h}$ and shunt capacitance $C_{h}$ are introduced by the horizontal part of the I-shaped slot; the series capacitance $C_{v}$ is caused by its vertical part. For simplicity, the capacitance introduced between the patch and the ground plane is included in the shunt capacitance $C_{h}$. Again, the series inductances $L_{t 1}$, $L_{t 2}$, and $L_{v}$ are introduced by the main patch.

With these additional elements, the transmission (ABCD) matrix of Unit B becomes:

$$
\begin{aligned}
{\left[\begin{array}{cc}
A & B \\
C & D
\end{array}\right] } & =\left[\begin{array}{cc}
1 & 0 \\
Y_{s} & 1
\end{array}\right] \times\left[\begin{array}{cc}
1 & Z_{t} \\
0 & 1
\end{array}\right] \times\left[\begin{array}{cc}
1 & 0 \\
Y_{h} & 1
\end{array}\right] \\
& \times\left[\begin{array}{cc}
1 & Z_{v} \\
0 & 1
\end{array}\right] \times\left[\begin{array}{cc}
1 & 0 \\
Y_{h} & 1
\end{array}\right] \times\left[\begin{array}{cc}
1 & Z_{t} \\
0 & 1
\end{array}\right] \\
& \times\left[\begin{array}{cc}
1 & 0 \\
Y_{s} & 1
\end{array}\right]
\end{aligned}
$$

where element admittances and impedances are

$$
\begin{aligned}
Y_{s} & =j \omega C_{s}+\frac{1}{j \omega L_{s}}, \quad Z_{t}=j \omega L_{t} \\
Y_{h} & =j \omega C_{h}+\frac{1}{j \omega L_{h}}, \quad Z_{v}=1 /\left(j \omega C_{v}+\frac{1}{j \omega L_{v}}\right)
\end{aligned}
$$


TABLE I

OPTIMIZED DESIGN PARAMETERS OF THE I-SHAPED SLOT-LOADED SIW LWA WITH PRW VIAS

\begin{tabular}{ccccccccc}
\hline \hline Parameter & $l_{s}$ & $W_{a}$ & $W_{b}$ & $L_{t}$ & $S$ & $d_{1}$ & $d_{2}$ & $P$ \\
\hline Value $(\mathrm{mm})$ & 82.8 & 1.5 & 7 & 5 & 1.2 & 0.5 & 0.8 & 10.8 \\
\hline
\end{tabular}

By solving (3) and (7), the dispersion relation can be expressed in terms of the wave number as:

$$
\begin{aligned}
\beta= & \frac{1}{P} \cos ^{-1}\left[Z_{t} Z_{v} Y_{s} Y_{h}\left(Z_{t} Y_{h}+2\right)+Z_{t} Y_{h}\left(2 Z_{t} Y_{s}\right.\right. \\
& \left.\left.+Z_{v} Y_{h}\right)+\left(2 Z_{t}+Z_{v}\right)\left(Y_{s}+Y_{h}\right)+1\right]
\end{aligned}
$$

To realize continuous beam scanning, the operating mode of any periodic SIW LWA should be a higher harmonic, such as $n=-1$ harmonic. The optimized dimensions for the Ishaped slot (in millimeters) are: $l_{h}=6, l_{v}=4, w_{s}=1$. The corresponding updated circuit parameters are: $L_{t}=0.52 \mathrm{nH}$, $L_{h}=0.15 \mathrm{nH}, C_{h}=0.6 \mathrm{pF}, L_{v}=0.53 \mathrm{pF}$, and $C_{v}=1 \mathrm{pF}$, while $L_{s}$ and $C_{s}$ remain the same $\left(L_{s}=0.3 \mathrm{nH}, C_{s}=1 \mathrm{pF}\right)$. Substituting these circuit parameter values into (8) and (9), the resulting dispersion relation of the $n=-1$ mode dispersion of Unit B is shown in Fig. 4 and labeled as Equivalent circuit. The corresponding dispersion curve obtained from the eigenmode solver is also shown in Fig. 4 and labeled as Eigenmode simulation. Both curves agree reasonably well in Unit B's leaky wave region. One observes that there is a continuous transition between the negative and positive regions of the dispersion curves at $10.9 \mathrm{GHz}$, i.e., the matched condition has been satisfied. Consequently, Unit B has no OSB. This feature appears in contrast to Unit A because the etched Ishaped slot and the PRW vias co-excite the higher harmonic $n=-1$ mode. Therefore, the Unit B-based SIW LWA can realize continuous beam scanning through broadside.

\section{I-SHAPED SLOT-LOADED SIW LWA WITH PRW VIAS}

The continuous beam scanning and CP performance of the I-shaped slot-loaded SIW LWA with PRW vias is analyzed. Details of the antenna configuration are given. The resulting continuous beam scanning and $\mathrm{CP}$ features are simulated.

\section{A. Antenna Configuration}

An I-shaped slot-loaded SIW LWA with PRW vias is developed with six Unit B cells to illustrate its performance characteristics. Fig. 5 shows the top view of the entire antenna configuration. Its optimized dimensions (in millimeters) are summarized in Table I. It is also constructed with the aforementioned two-layer copper-clad F4BM-2 substrate. Two tapered pads, tapering from a width of $w_{a}$ to a width of $w_{b}$ over the length of $l_{t}$, are used to connect the $50 \Omega$ feed line to the main patch over a wide bandwidth. The antenna is fed from the left end and terminated on the right end with a matching $50 \Omega$ load.

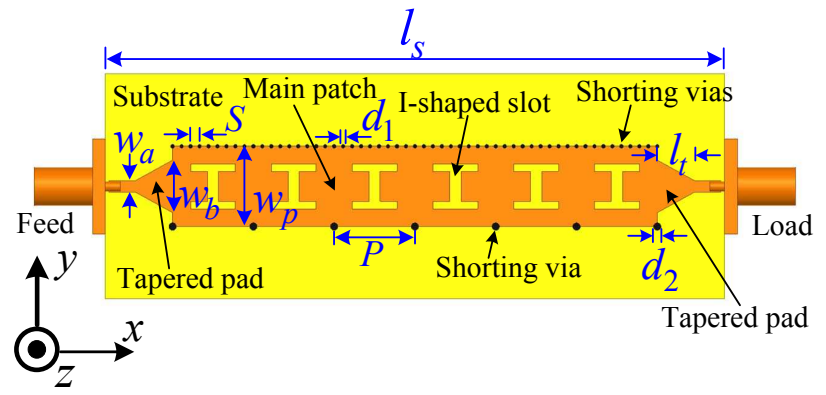

Fig. 5. I-shaped slot-loaded SIW LWA with PRW vias configuration.

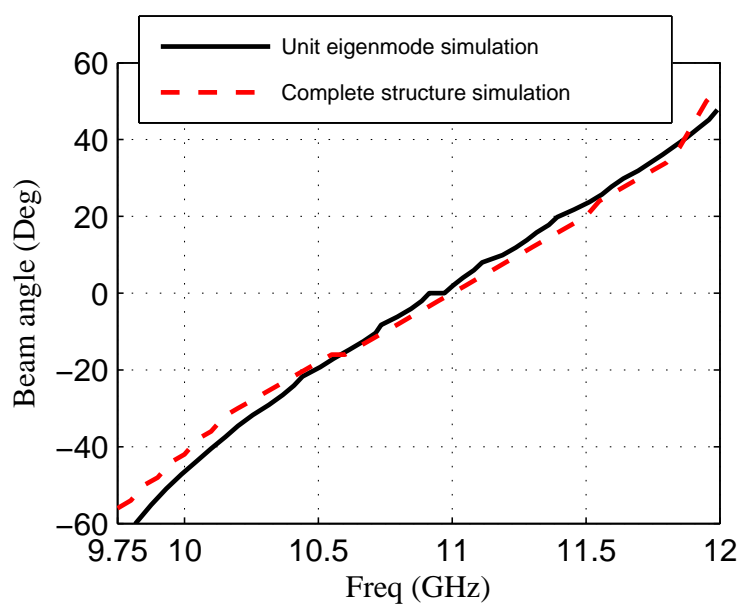

Fig. 6. Main beam angle when the source frequency is varied as calculated from the simulations of the eigenmode (infinite) and complete (finite) structure.

\section{B. Beam Scanning}

The main beam angle accomplished through the above complete six-cell system simulation is shown in Fig. 6, and the results are labeled as Complete structure simulation. Based on the dispersion properties obtained from the eigenmode simulation shown in Fig. 4, the main beam angles can be calculated using (1), and the corresponding results are labeled as Unit eigenmode simulation in Fig. 6 for comparison. It is seen that both results agree very well. The minor discrepancies arise from the difference between the finite length of the complete structure and the infinite length of the eigenmodebased results. Note that when the source frequency is swept from 9.75 to $12.0 \mathrm{GHz}$, the main beam of the finite structure is continuously scanned from $-56^{\circ}$ to $+54^{\circ}$, through $110^{\circ}$.

The simulated directivity and AR values of the six-cell system are presented in Fig. 7. The directivity values vary between 6.8 and $11.5 \mathrm{dBic}$. The AR values are greater than $3 \mathrm{~dB}$ across the operating band except for a very narrow frequency range around $11.1 \mathrm{GHz}$ where the $\mathrm{AR}$ is $\leq 3 \mathrm{~dB}$.

\section{Circular Polarization Analysis}

To analyze the CP performance of the six-cell system, one can introduce its equivalent electric and magnetic current sources. According to Huygens' principle [34], the antenna's far-field radiation can be determined from these equivalent sources. Fig. 8 shows the simulated electric and magnetic 


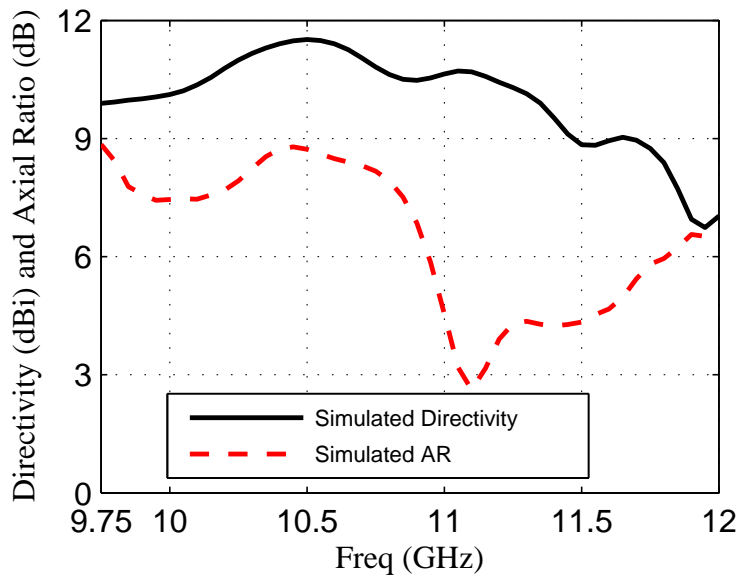

Fig. 7. Simulated directivity and axial ratio values of the six-cell I-shaped slot-loaded SIW LWA with PRW vias.

fields just above one Unit $\mathrm{B}$ in the system in Fig. 3 at $t=0$ and $\pi / 4$ for $10.5 \mathrm{GHz}$. It is seen that the magnetic fields are very small and negligible in comparison to the electric fields. Consequently, only these near-field electric fields are considered for the analysis of the far-field radiation performance. They are mainly distributed above the etched I-shaped slot and the PRW vias.

To emphasize this distribution more clearly, Fig. 9(a) shows the simulated E-field components $\mathbf{E}_{t}, \mathbf{E}_{m}, \mathbf{E}_{b}$, and $\mathbf{E}_{p r w}$ above one Unit B cell. As shown in Fig. 9 (b), the corresponding equivalent magnetic currents are calculated as:

$$
\begin{gathered}
\mathbf{M}_{t}=\mathbf{E}_{t} \times \hat{\mathbf{n}}_{z} ; \quad \mathbf{M}_{m}=\mathbf{E}_{m} \times \hat{\mathbf{n}}_{z} ; \\
\mathbf{M}_{b}=\mathbf{E}_{b} \times \hat{\mathbf{n}}_{z} ; \quad \mathbf{M}_{p r w}=-\mathbf{E}_{p r w} \times \hat{\mathbf{n}}_{y}
\end{gathered}
$$

where $\hat{\mathbf{n}}_{y}$, and $\hat{\mathbf{n}}_{z}$ are the unit vectors along the $+y$ axis and $+z$ axis, respectively. Note that the two horizontal magnetic currents $\mathbf{M}_{t}$ and $\mathbf{M}_{b}$ along the $x$-axis direction are introduced by the two horizontal parts of the etched I-shaped slot. Similarly, the horizontal magnetic current $\mathbf{M}_{p r w}$ along the $x$-axis direction is produced by the PRW vias. The vertical current $\mathbf{M}_{m}$ along the $y$-axis direction is introduced by the vertical part of the etched I-shaped slot. Then, the total equivalent magnetic source $\mathbf{M}_{\text {tot }}$ is:

$$
\mathbf{M}_{\mathrm{tot}}=\mathbf{M}_{\mathbf{x}}+\mathbf{M}_{\mathbf{y}}
$$

where

$$
\mathbf{M}_{\mathbf{x}}=\mathbf{M}_{t}+\mathbf{M}_{b}+\mathbf{M}_{p r w}, \quad \text { and } \quad \mathbf{M}_{\mathbf{y}}=\mathbf{M}_{m}
$$

It is obvious that to form a circularly polarized wave, the two magnetic current components, i.e. $\mathbf{M}_{\mathbf{x}}$ along the $x$-axis direction and $\mathbf{M}_{\mathbf{y}}$ along the $y$-axis direction, need to have the same amplitude and a $90^{\circ}$ phase difference. It is found for this six-cell I-shaped slot-loaded system that $\mathbf{M}_{p r w}$ is approximately out of phase with $\mathbf{M}_{b}$, and $\mathbf{M}_{t}$ is relatively small. As a consequence, $\mathbf{M}_{\mathbf{x}}$ is much smaller than $\mathbf{M}_{\mathbf{y}}$. Therefore, the noted poor AR values are observed. To improve the AR performance, a different unit cell had to be developed.

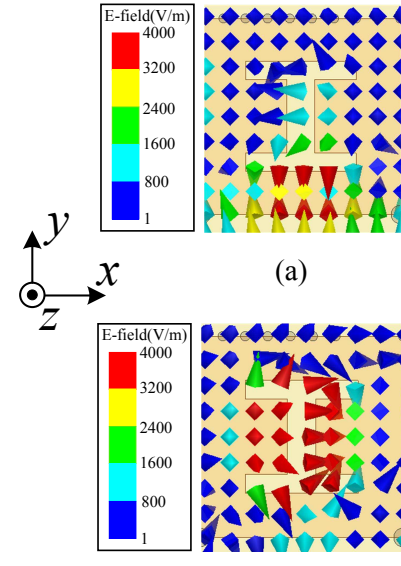

(c)

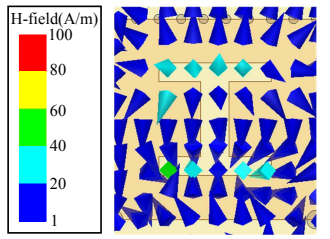

(b)
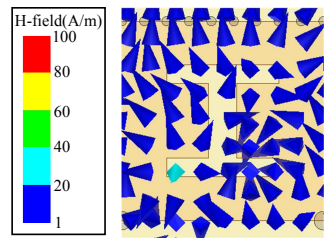

(d)
Fig. 8. Top view of the field distribution on the Unit B at $10.5 \mathrm{GHz}$. (a) E-fields distribution $(t=0)$ (b) H-field distribution $(t=0)$ (c) E-fields distribution $(t=\pi / 4)$ (d) H-fields distribution $(t=\pi / 4)$.
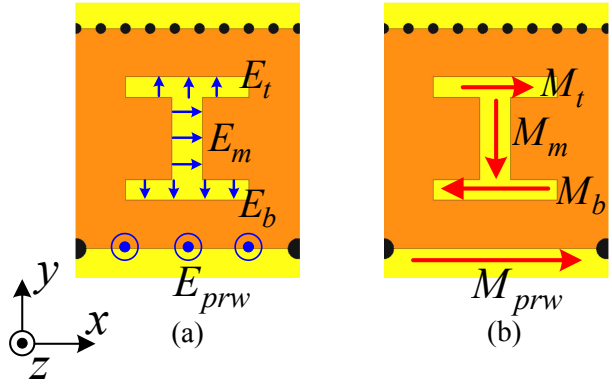

(b)

Fig. 9. Unit $\mathrm{B}$ radiation mechanism analysis. (a) E-fields distribution. (b) Equivalent magnetic current sources.

\section{BENZENE-RING-Shaped SLOT-LOADED SIW CP LWA WITH PRW VIAS}

The benzene-ring-shaped slot-loaded SIW CP LWA with PRW Vias is introduced to achieve much improved AR values. Its continuous beam scanning and $\mathrm{CP}$ performance is demonstrated. Details of the antenna configuration are given. The resulting continuous beam scanning and $\mathrm{CP}$ features are analyzed and simulated.

\section{A. Antenna Configuration}

Guided by the field distribution analysis of the I-slots, it was found that one can manipulate the etched slots and optimize the PRW vias to improve the AR values. In particular, a new slot-loaded unit cell was developed. It is labelled as Unit C in the top right inset of Fig. 10(a). Its evolution from Unit B with the I-slot is depicted in the three subplots of Fig. 10(a). The top and bottom horizontal parts of the I-shaped slot were first rotated to an angle of $\psi\left(\psi=45^{\circ}\right)$. This modification reduced the magnetic current contribution $\mathbf{M}_{b}$ found in Fig. 9. These modified slots were then connected to the counterparts in the adjacent cells using additional horizontal slots on the upper side of the patch. These additional horizontal slots add another $x$-axis magnetic current component to balance $\mathbf{M}_{x}$ and $\mathbf{M}_{y}$. The final shape is reminiscent of a benzene ring. Furthermore, by cascading these benzene-ring-shaped slot unit 


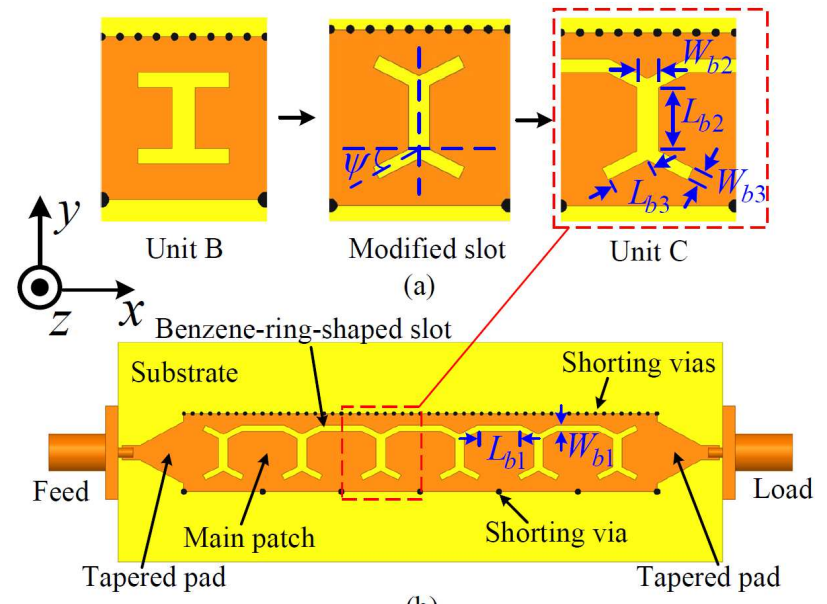

(b)

Fig. 10. Benzene-ring-shaped slot-loaded SIW CP LWA with PRW vias. (a) Evolution of Unit C from Unit B. (b) Complete six-cell based structure.

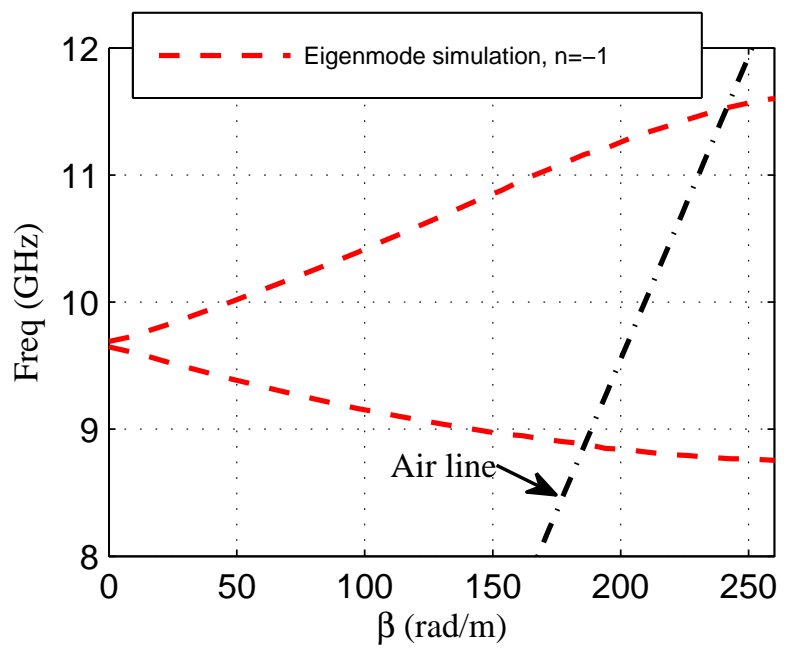

Fig. 11. Unit $\mathrm{C}$ dispersion diagrams obtained from its eigenmode simulation.

cells, it was found that their longer length slots enhance the guiding characteristics of the traveling wave in the SIW structure in comparison to their resonant I-shaped parent.

The dispersion diagram of the Unit $\mathrm{C}$ shown in Fig. 11 was obtained with an eigenmode simulation. It indicates that a continuous transition between the backward and forward curves is achieved. Six benzene-ring-shaped slot-loaded Unit C cells were then combined with the tapered feed and termination segments to form the SIW LWA with PRW vias shown in Fig. 10(b). The dimensions of the benzene-ring-shaped slots are represented by the parameters $L_{b 1}, W_{b 1}, L_{b 2}, W_{b 2}, L_{b 3}$, and $W_{b 3}$ in Fig. 10.

\section{B. Design Parameter Study}

With the knowledge that the $\mathrm{CP}$ performance of a slotloaded SIW LWA with PRW vias is dependent on the precise shape of its etched slots and the PRW vias, the design parameters of the benzene-ring-shaped slot and PRW vias combination were carefully investigated and optimized. In particular, parameter studies of the slot widths $W_{b 1}, W_{b 2}$, and

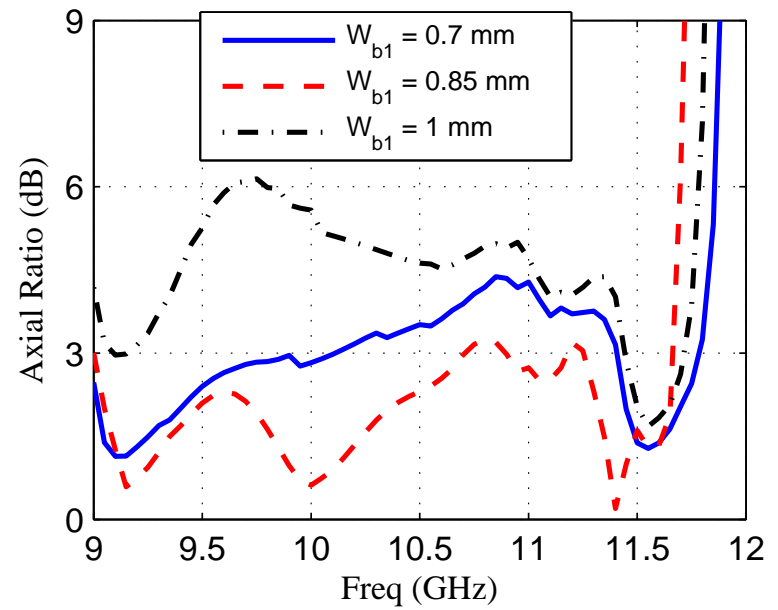

Fig. 12. The axial ratio (AR) values as a function of the source frequency for different values of $W_{b 1}$.

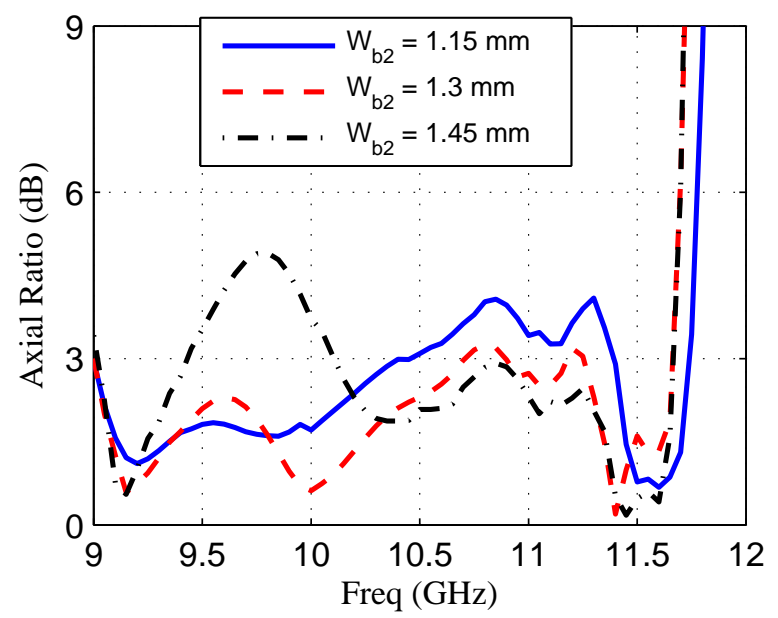

Fig. 13. The axial ratio (AR) values as a function of the source frequency for different values of $W_{b 2}$.

the PRW via radius $d_{2}$ were performed. When the value of the studied parameter was changed, the values for the other parameters were fixed.

Fig. 12 shows the AR values as a function of the source frequency for different values of $W_{b 1}$. It is seen that when $W_{b 1}=0.7 \mathrm{~mm}$, the AR values exceed $3 \mathrm{~dB}$ between 10.1 and $11.4 \mathrm{GHz}$. However, when the value of $W_{b 1}$ increases to $0.85 \mathrm{~mm}$, good CP performance below $3.1 \mathrm{~dB}$ is obtained within the whole operating band from 9 to $11.5 \mathrm{GHz}$. With a further increase of $W_{b 1}$ to $1.0 \mathrm{~mm}$, the overall CP performance again deteriorates, the $\mathrm{AR}$ values of $\mathrm{AR}$ being more than 3 $\mathrm{dB}$. Consequently, it is seen that $W_{b 1}$ has a high impact on the antenna's CP performance.

Fig. 13 shows the AR values as a function of the source frequency for different values of $W_{b 2}$. Good CP performance is obtained for $W_{b 2}=1.3 \mathrm{~mm}$. In contrast, the AR values in the higher frequency band from 10.5 to $11.4 \mathrm{GHz}$ becomes worse when the value of $W_{b 2}$ reduces to $1.15 \mathrm{~mm}$. Similarly, the AR values in the lower frequency band from 9.45 to $10.1 \mathrm{GHz}$ 


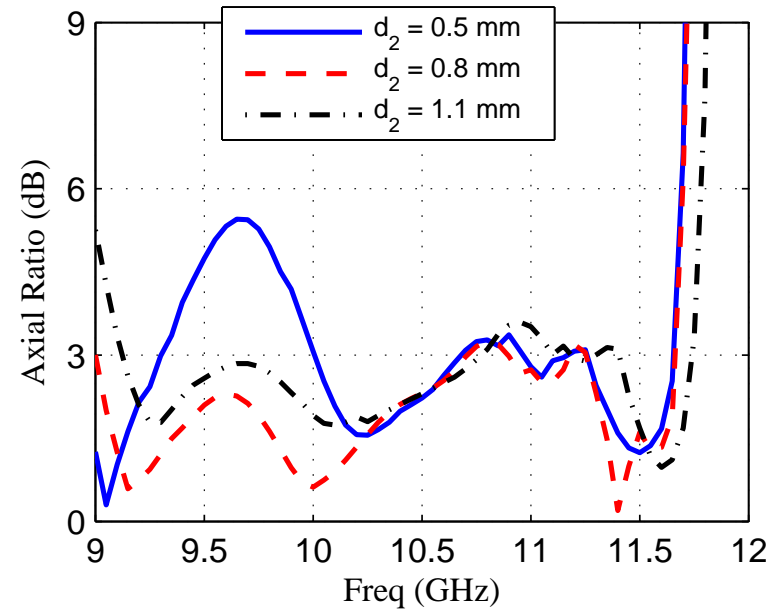

Fig. 14. The axial ratio (AR) as a function of the source frequency for different values of $d_{2}$.

TABLE II

OPTIMIZED VALUES OF THE DESIGN PARAMETERS OF THE BENZENE-RING-SHAPED SLOT-LOADED SIW CP LWA WITH PRW VIAS

\begin{tabular}{cccccccc}
\hline \hline Parameter & $l_{s}$ & $W_{a}$ & $W_{b}$ & $L_{t}$ & $S$ & $d_{1}$ & $d_{2}$ \\
\hline Value $(\mathrm{mm})$ & 82.8 & 2 & 8.5 & 6 & 1.2 & 0.5 & 0.8 \\
\hline Parameter & $P$ & $L_{b 1}$ & $W_{b 1}$ & $L_{b 2}$ & $W_{b 2}$ & $L_{b 3}$ & $W_{b 3}$ \\
\hline Value $(\mathrm{mm})$ & 10.8 & 5.75 & 0.85 & 3.9 & 1.3 & 2.7 & 0.8 \\
\hline
\end{tabular}

deteriorate when the value of $W_{b 2}$ increases to $1.45 \mathrm{~mm}$. Thus, $W_{b 2}=1.3 \mathrm{~mm}$ was chosen to balance the CP performance in both the lower and higher frequency bands and yielded good $\mathrm{CP}$ performance over the entire operating band from 9 to 11.5 $\mathrm{GHz}$.

Finally, Fig. 14 shows the AR values as a function of the source frequency for different values of $d_{2}$. It is seen that when $d_{2}=0.5 \mathrm{~mm}$, the AR values remain below $3.2 \mathrm{~dB}$ except in the lower frequency band from 9.3 to $10 \mathrm{GHz}$. When $d_{2}$ increases to $0.8 \mathrm{~mm}$, the AR performance is improved and the AR values are all below $3.1 \mathrm{~dB}$. When $d_{2}$ further increases to $1.1 \mathrm{~mm}$, the AR performance remains similar, but the operating band blue shifts to higher frequencies.

To observe the quadrature phase between the two main E-field components, two observation points were selected to extract each of their phase values. One was located at the center point of a slot and the other was located on the open edge at the center point of the two adjacent vias. The phase differences of the two E-field components at these points were found to be between $78^{\circ}$ and $108^{\circ}$ over the operating frequency band. Thus, they were close to the $90^{\circ}$ value associated with the $\mathrm{CP}$ performance. The benzene-ring-shaped slot parameters $W_{b 1}, W_{b 2}$, and $d_{2}$ are the ones that mainly affected those phase differences.

The final optimized parameter values are listed in Table II.

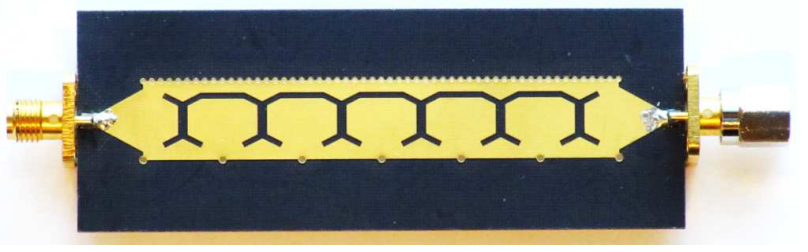

Fig. 15. Photograph of the fabricated benzene-ring-shaped slot-loaded CP SIW LWA with PRW vias prototype.

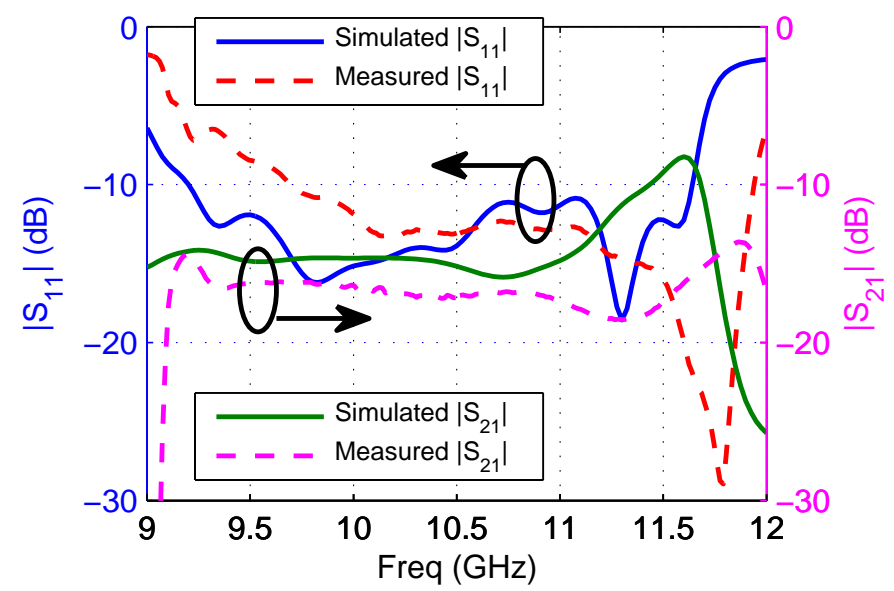

Fig. 16. Simulated and measured S-parameters of the benzene-ring-shaped slot-loaded CP SIW LWA with PRW vias.

\section{Simulated and Measured Results}

Based on the optimum design parameter values, the benzene-ring-shaped slot-loaded CP SIW LWA with PRW vias was fabricated and measured. Fig. 15 shows a photograph of the fabricated prototype. The simulated and measured Sparameters are compared Fig. 16. It is seen that the simulated $\left|S_{11}\right| \leq-10 \mathrm{~dB}$ operating bandwidth is from 9.2 to 11.65 $\mathrm{GHz}$. When the source frequency is reduces from 9.3 down to $9.0 \mathrm{GHz}$, the simulated $\left|S_{11}\right|$ value remains below $-6.4 \mathrm{~dB}$. The measured $\left|S_{11}\right| \leq-10$ operating bandwidth is from 9.7 to $11.94 \mathrm{GHz}$. For frequencies between 9.35 and $9.7 \mathrm{GHz}$, the measured $\left|S_{11}\right|$ is $\leq-6.6 \mathrm{~dB}$. Noted that the measured $\left|S_{11}\right|$ values show a blue shift to higher operating frequencies. Simulations indicate that this is caused by fabrication inaccuracies. The simulated $\left|S_{21}\right|$ values are below $-10 \mathrm{~dB}$ for frequencies lower than $11.45 \mathrm{GHz}$. They peak at 11.6 $\mathrm{GHz}$ with the value of $-8.23 \mathrm{~dB}$. The measured $\left|S_{21}\right|$ values are always below $-13.6 \mathrm{~dB}$. The simultaneously measured low $\left|S_{11}\right|$ and $\left|S_{21}\right|$ values indicate that the system has good radiation performance.

Fig. 17 displays the simulated radiation patterns at five different frequencies. It illustrates that the main beam can be continuously scanned from backward to forward directions through broadside. The main beam points in the backward direction at $9 \mathrm{GHz}$ with the leftmost angle of $-46^{\circ}$. With the increase in the source frequency, the beam moves towards the broadside. For instance, the main beam angle is $-26^{\circ}$ at $9.25 \mathrm{GHz}$. The main beam points in the broadside direction at 9.8 GHz. When the source frequency is further increased, the 


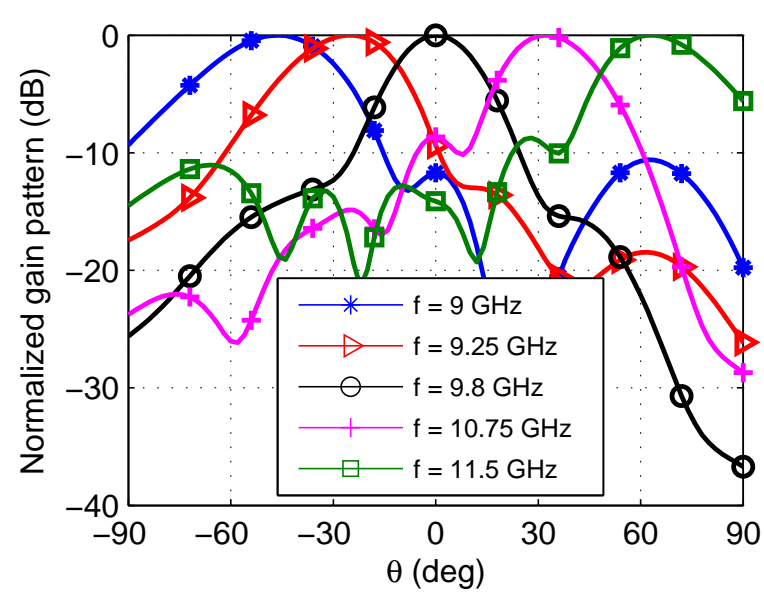

Fig. 17. Simulated radiation patterns of the optimized design at five different frequencies.

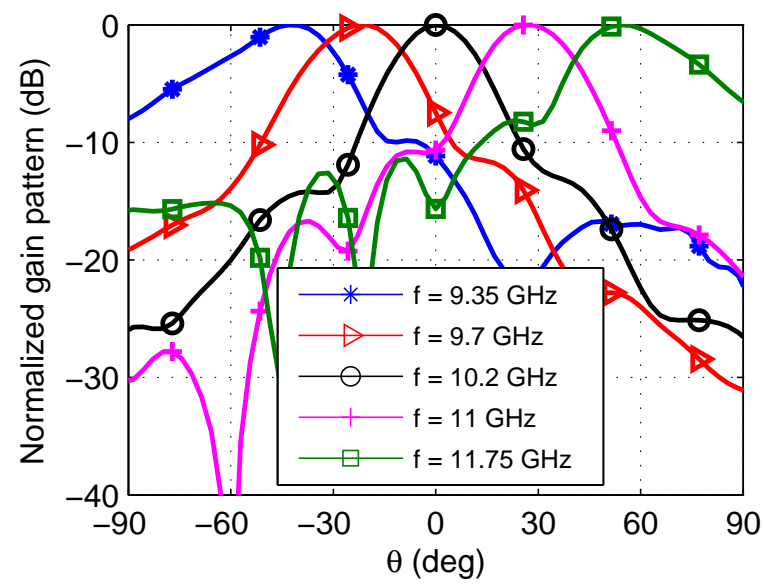

Fig. 18. Measured radiation patterns of the prototype at five different frequencies.

main beam moves into the forward directions, e.g., the main beam angle is $+32^{\circ}$ at $10.75 \mathrm{GHz}$. The main beam points at the rightmost angle $\left(+64^{\circ}\right)$ at $11.5 \mathrm{GHz}$. Thus, the simulated beam scanning range is $110^{\circ}$ as the source frequency is swept from 9 to $11.5 \mathrm{GHz}$. The simulated radiation efficiency of the antenna varies from $92.3 \%$ to $97.9 \%$ within the operating frequency band, 9.0 to $11.5 \mathrm{GHz}$.

The far-field radiation patterns of the prototype were measured using the Satimo multi-probe spherical near-field system StarLab-18 located at Xiamen University, China. The measured radiation patterns at five different frequencies are shown in Fig. 18. When the operating frequency is 9.35, 9.7, 10.2, $11,11.75 \mathrm{GHz}$, the main beam angle is $-42.8^{\circ},-22.8^{\circ}$, $0^{\circ},+25.7^{\circ}$, and $+54.3^{\circ}$, respectively. The measured beam scanning range is $97.1^{\circ}$ as the source frequency varies from 9.35 to $11.75 \mathrm{GHz}$. The corresponding simulated (measured) cross-pol levels in the main beam directions at all of these frequencies are all smaller than $-15.1(-12.7) \mathrm{dB}$.

Fig. 19 compares the simulated and measured AR values along with the realized gain values as functions of the source frequency. As noted previously, the simulated AR values are

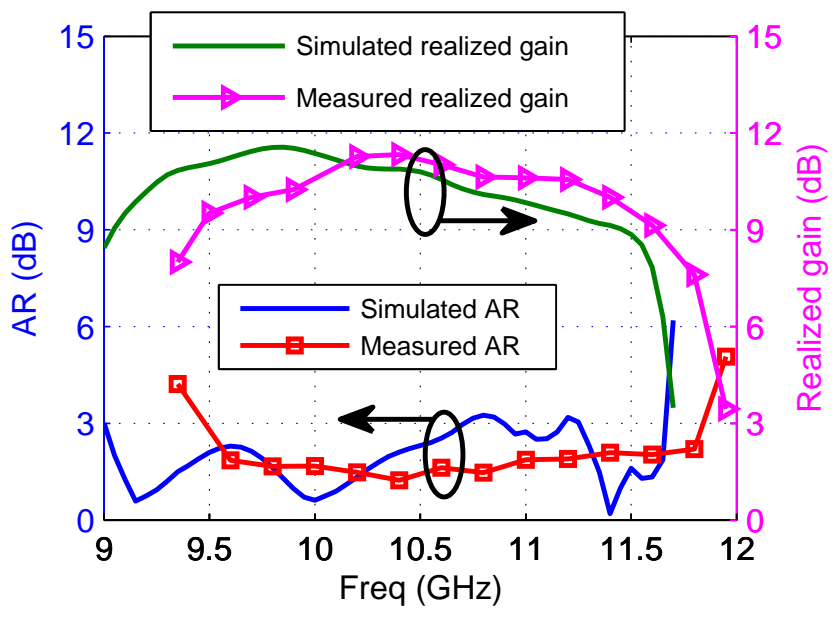

Fig. 19. Simulated and measured axial ratio (AR) values together with the corresponding realized gain values as functions of the source frequency.

below $3.1 \mathrm{~dB}$ within the operational frequency range from 9 to $11.5 \mathrm{GHz}$. The corresponding realized gain values vary between 8.44 and $11.56 \mathrm{dBic}$. Due to the shift of the measured results to higher frequencies, the AR values below $3 \mathrm{~dB}$ occur in the band from 9.45 to $11.75 \mathrm{GHz}$. The AR value below this band at $9.35 \mathrm{GHz}$, for example, is $4.1 \mathrm{~dB}$. From 9.35 to 11.75 $\mathrm{GHz}$, the measured realized gain is stable with only a $3.3 \mathrm{~dB}$ gain variation between 8 and $11.3 \mathrm{dBic}$.

\section{DISCUSSION}

Considering its potential applications, the current study was focused on achieving a LWA system that realizes wideangle CP beam scanning with a consistent gain. While there are examples of systems that can scan a CP beam over a large angular range, it generally remains a very challenging problem. Our goal was achieved by introducing a novel unit cell with a benzene-ring-shaped slot and using it to construct a six-cell SIW LWA with PRW vias. Continuous beam scanning in a wide angular range with consistent gain was realized with an optimized design. A complete comparison between this optimized design and previously reported CP LWAs is given in Table III. It is seen that when it is compared to these reference LWAs, the antenna system developed in this paper exhibits superior performance in terms of its beam scanning range and its realized gain consistency.

\section{CONCLUSION}

A benzene-ring-shaped slot-loaded CP SIW LWA with PRW vias was presented. It was demonstrated that it realizes continuous beam scanning from its backward to forward directions through a wide angular range with consistent realized gain. It was shown that an I-shaped slot-loaded LWA with PRW vias supports continuous beam scanning, but the AR values in the main beam directions are very high. To achieve the $\mathrm{CP}$ beam scanning in a wide angle range with consistent gain, a novel benzene-ring-shaped slot-loaded SIW LWA with PRW vias was developed and optimized. A prototype system was fabricated and tested. The measured results demonstrate 
TABLE III

COMPARISON BETWEEN THE ANTENNA DEVELOPED IN THIS PAPER AND THE REPORTED CP LWAS

\begin{tabular}{c|c|c|c|c|c|c}
\hline \hline Antenna Ref. & Antenna type & Operating band & Antenna length & AR $\leq 3 \mathrm{~dB}$ band & Beam scanning range & Gain range \\
\hline$[19]$ & Inspired-CRLH SIW (Single-layer) & $4.2-4.85 \mathrm{GHz}$ & $2.43 \lambda_{0}$ & $4.2-4.85 \mathrm{GHz}$ & $-25^{\circ}$ to $+26^{\circ}$ & -1 to $1.5 \mathrm{dBic}$ \\
\hline$[20]$ & CRLH microstrip (Single-layer) & $23-25 \mathrm{GHz}$ & $22.0 \lambda_{0}$ & Not given & $-6^{\circ}$ to $+6^{\circ}$ & 15 to $16 \mathrm{dBic}$ \\
\hline$[21]$ & CRLH HMSIW (Single-layer) & $7.4-13.5 \mathrm{GHz}$ & $6.09 \lambda_{0}$ & Not given & $-70^{\circ}$ to $+70^{\circ}$ & 7.48 to $12.01 \mathrm{dBic}$ \\
\hline$[22]$ & CRLH SIW (Single-layer) & $7.35-10.15 \mathrm{GHz}$ & $4.56 \lambda_{0}$ & $7.5-9.25 \mathrm{GHz}$ & $-19^{\circ}$ to $+84^{\circ}$ & 0 to $8.95 \mathrm{dBic}$ \\
\hline$[23]$ & SSPP TL (Double-layer) & $11-15 \mathrm{GHz}$ & $5.72 \lambda_{0}$ & Not given & $-32^{\circ}$ to $+34^{\circ}$ & 12.8 to $14.2 \mathrm{dBic}$ \\
\hline$[28]$ & Slot-loaded SIW (Single-layer) & $10-14 \mathrm{GHz}$ & $6.4 \lambda_{0}$ & $10-13.8 \mathrm{GHz}$ & $-40^{\circ}$ to $+25^{\circ}$ & 4.2 to $11 \mathrm{dBic}$ \\
\hline This work & Slot-loaded SIW with PRW (Single-layer) & $9.35-11.75 \mathrm{GHz}$ & $2.91 \lambda_{0}$ & $9.45-11.75 \mathrm{GHz}$ & $-42.8^{\circ}$ to $+54.3^{\circ}$ & 8 to $11.3 \mathrm{dBic}$ \\
\hline
\end{tabular}

that the beam radiated by this system can be scanned from $-42.8^{\circ}$ to $+54.3^{\circ}$, i.e., continuous beam scanning through $97.1^{\circ}$ ) was realized, and it has a commensurate gain variation of $3.3 \mathrm{dBic}$ between 8 and $11.3 \mathrm{dBic}$. The reported $\mathrm{CP}$ LWA is a highly promising candidate for future wireless communications systems for which there exists a prominent demand for high performance antennas that are low cost and exhibit multiple functionality.

\section{ACKNOWLEDGMENT}

The authors would like to thank Mr. Ming Li from Xiamen University in China for his assistance in the measurement campaign.

\section{REFERENCES}

[1] A. A. Oliner, "Leakage from higher modes on microstrip line with application to antennas," Radio Science, vol. 22, no. 6, pp. 907-912, 1987.

[2] D. R. Jackson, C. Caloz, and T. Itoh, "Leaky-wave antennas," Proc. IEEE, vol. 100, no. 7, pp. 2194-2206, Jul. 2012.

[3] C. Jin, A. Alphones, and M. Tsutsumi, "Double periodic composite right/left handed transmission line and its applications to compact leakywave antennas," IEEE Trans. Antennas Propag., vol. 59, no. 10, pp. 3679-3686, Oct. 2011.

[4] Y. Dong and T. Itoh, "Substrate integrated composite right-/left-handed leaky-wave structure for polarization-flexible antenna application," IEEE Trans. Antennas Propag., vol. 60, no. 2, pp. 760-771, Feb. 2012.

[5] A. J. Martinez-Ros, J. L. Gomez-Tornero, and G. Goussetis, "Planar leaky-wave antenna with flexible control of the complex propagation constant," IEEE Trans. Antennas Propag., vol. 60, no. 3, pp. 1625-1630, Mar. 2012.

[6] Z. Li, J. Wang, M. Chen, and Z. Zhang, "New approach of radiation pattern control for leaky-wave antennas based on the effective radiation sections," IEEE Trans. Antennas Propag., vol. 63, no. 7, pp. 2867-2878, Jul. 2015.

[7] N. Nguyen-Trong, L. Hall, and C. Fumeaux, "Transmission-line model of nonuniform leaky-wave antennas," IEEE Trans. Antennas Propag., vol. 64, no. 3, pp. 883-893, Mar. 2016.

[8] T. K. Nguyen, H. H. Tran, and N. Nguyen-Trong, "A wideband dualcavity-backed circularly polarized crossed dipole antenna," IEEE Antennas Wireless Propag. Lett., vol. 16, pp. 3135-3138, 2017.

[9] W. Lin and H. Wong, "Wideband circular polarization reconfigurable antenna," IEEE Trans. Antennas Propag., vol. 63, no. 12, pp. 59385944, Dec. 2015.

[10] W. Lin, S. L. Chen, R. W. Ziolkowski, and Y. J. Guo, "Reconfigurable, wideband, low-profile, circularly polarized antenna and array enabled by an artificial magnetic conductor ground," IEEE Trans. Antennas Propag., vol. 66 , no. 3, pp. 1564-1569, Mar. 2018
[11] S. Paulotto, P. Baccarelli, F. Frezza, and D. R. Jackson, "Full-wave modal dispersion analysis and broadside optimization for a class of microstrip CRLH leaky-wave antennas," IEEE Trans. Microw. Theory Techn., vol. 56, no. 12, pp. 2826-2837, Dec. 2008.

[12] Y. Li, Q. Xue, E. K.-N. Yung, and Y. Long, "The periodic half-width microstrip leaky-wave antenna with a backward to forward scanning capability," IEEE Trans. Antennas Propag., vol. 58, no. 3, pp. 963-966, Mar. 2010.

[13] D. K. Karmokar and K. P. Esselle, "Periodic U-slot-loaded dual-band half-width microstrip leaky-wave antennas for forward and backward beam scanning," IEEE Trans. Antennas Propag., vol. 63, no. 12, pp. 5372-5381, Dec. 2015.

[14] M. H. Rahmani and D. Deslandes, "Backward to forward scanning periodic leaky-wave antenna with wide scanning range," IEEE Trans. Antennas Propag., vol. 65, no. 7, pp. 3326-3335, Jul. 2017.

[15] G. Minatti, F. Caminita, M. Casaletti, and S. Maci, "Spiral leaky-wave antennas based on modulated surface impedance," IEEE Trans. Antennas Propag., vol. 59, no. 12, pp. 4436-4444, Dec. 2011

[16] A. Lai, T. Itoh, and C. Caloz, "Composite right/left-handed transmission line metamaterials," IEEE Microwave Mag., vol. 5, no. 3, pp. 34-50, Sep. 2004

[17] Nasimuddin, Z. N. Chen, and X. Qing, "Multilayered composite right/left-handed leaky-wave antenna with consistent gain," IEEE Trans. Antennas Propag., vol. 60, no. 11, pp. 5056-5062, Nov. 2012.

[18] — "Substrate integrated metamaterial-based leaky-wave antenna with improved boresight radiation bandwidth," IEEE Trans. Antennas Propag., vol. 61, no. 7, pp. 3451-3457, Jul. 2013.

[19] H. Lee, J. H. Choi, C. T. M. Wu, and T. Itoh, "A compact single radiator CRLH-inspired circularly polarized leaky-wave antenna based on substrate-integrated waveguide," IEEE Trans. Antennas Propag. vol. 63 , no. 10 , pp. $4566-4572$, Oct. 2015.

[20] S. Otto, Z. Chen, A. Al-Bassam, A. Rennings, K. Solbach, and C. Caloz, "Circular polarization of periodic leaky-wave antennas with axial asymmetry: Theoretical proof and experimental demonstration," IEEE Trans. Antennas Propag., vol. 62, no. 4, pp. 1817-1829, Apr. 2014.

[21] A. P. Saghati, M. M. Mirsalehi, and M. H. Neshati, "A hmsiw circularly polarized leaky-wave antenna with backward, broadside, and forward radiation," IEEE Antennas Wireless Propag. Lett., vol. 13, pp. 451-454, 2014.

[22] M. M. Sabahi, A. A. Heidari, and M. Movahhedi, "A compact CRLH circularly polarized leaky-wave antenna based on substrate-integrated waveguide," IEEE Trans. Antennas Propag., vol. 66, no. 9, pp. 44074414, Sep. 2018.

[23] D. F. Guan, P. You, Q. Zhang, Z. H. Lu, S. W. Yong, and K. Xiao, "A wide-angle and circularly polarized beam-scanning antenna based on microstrip spoof surface plasmon polariton transmission line," IEEE Antennas Wireless Propag. Lett., vol. 16, pp. 2538-2541, 2017.

[24] Q. Zhang, Q. Zhang, and Y. Chen, "High-efficiency circularly polarised leaky-wave antenna fed by spoof surface plasmon polaritons," IET Microw. Antennas Propag., vol. 12, no. 10, pp. 1639-1644, 2018.

[25] S. Paulotto, P. Baccarelli, F. Frezza, and D. R. Jackson, "A novel technique for open-stopband suppression in 1-D periodic printed leakywave antennas," IEEE Trans. Antennas Propag., vol. 57, no. 7, pp. 1894 1906, Jul. 2009.

[26] J. Liu, D. R. Jackson, and Y. Long, "Substrate integrated waveguide 
(SIW) leaky-wave antenna with transverse slots," IEEE Trans. Antennas Propag., vol. 60, no. 1, pp. 20-29, Jan. 2012.

[27] J. Liu, X. Tang, Y. Li, and Y. Long, "Substrate integrated waveguide leaky-wave antenna with $\mathrm{H}$-shaped slots," IEEE Trans. Antennas Propag., vol. 60, no. 8, pp. 3962-3967, Aug. 2012.

[28] Y. L. Lyu, F. Y. Meng, G. H. Yang, D. Erni, Q. Wu, and K. Wu, "Periodic SIW leaky-wave antenna with large circularly polarized beam scanning range," IEEE Antennas Wireless Propag. Lett., vol. 16, pp. 2493-2496, 2017.

[29] C. Caloz and T. Itoh, Electromagnetic Metamaterials:Transmission Line Theory and Microwave Applications. Wiley-IEEE Press, 2006, pp. 347-352.

[30] Y. L. Lyu, X. X. Liu, P. Y. Wang, D. Erni, Q. Wu, C. Wang, N. Y. Kim, and F. Y. Meng, "Leaky-wave antennas based on noncutoff substrate integrated waveguide supporting beam scanning from backward to forward," IEEE Trans. Antennas Propag., vol. 64, no. 6, pp. 2155-2164, Jun. 2016.

[31] H. Mosallaei and K. Sarabandi, "Design and modeling of patch antenna printed on magneto-dielectric embedded-circuit metasubstrate," IEEE Trans. Antennas Propag., vol. 55, no. 1, pp. 45-52, Jan. 2007.

[32] H. W. Johnson, M. Graham et al., High-speed digital design: a handbook of black magic. Prentice Hall Upper Saddle River, NJ, 1993, vol. 1.

[33] D. K. Karmokar, Y. J. Guo, P. Y. Qin, S. L. Chen, and T. S. Bird, "Substrate integrated waveguide-based periodic backward-to-forward scanning leaky-wave antenna with low cross-polarization," IEEE Trans. Antennas Propag., vol. 66, no. 8, pp. 3846-3856, Aug. 2018.

[34] C. A. Balanis, Antenna Theory: Analysis and Design. John Willey \& Sons, 1997.

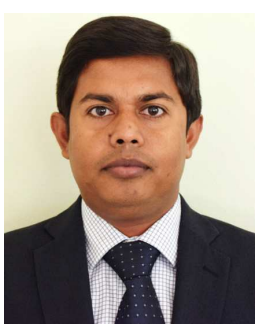

Debabrata K. Karmokar (S'12-M'15) received the B.Sc. degree in electrical \& electronic engineering (EEE) from the Khulna University of Engineering \& Technology (KUET), Khulna, Bangladesh, in 2007 and the $\mathrm{PhD}$ degree in Electronic Engineering from Macquarie University, Sydney, NSW, Australia in 2015. He was an Assistant Professor and a member of CRTS in the department of EEE, KUET, and an Assistant Director of Students' Welfare of the University. He was a Research Assistant, a Casual Academic, and also a Secretary of the IEEE Student Branch, at Macquarie University. He is currently working as a Postdoctoral Research Associate with the Global Big Data Technologies Centre (GBDTC), Faculty of Engineering and Information Technology, University of Technology Sydney (UTS), Ultimo, NSW, Australia.

Dr. Karmokar is a recipient of several scholarships and awards including a District Council Scholarship from Satkhira District Council, Bangladesh, Technical Scholarship from KUET, a Commonwealth-funded International Postgraduate Research Scholarship (IPRS) together with an International Macquarie University Research Excellence Scholarship (iMQRES), an OCE $\mathrm{PhD}$ Scholarship from the Commonwealth Scientific and Industrial Research Organisation (CSIRO) ICT Centre, Marsfield, Australia, the First Prize in the Poster Competition at the Engineering Symposium 2015, Macquarie University, Young Scientist Award from Venus International Foundation in 2018, and URSI Young Scientist Award in 2019. He has authored or coauthored of a book chapter and over 70 journal and conference papers. He is serving as a reviewer for several journals of IEEE, IET, Wiley, Springer and Elsevier.

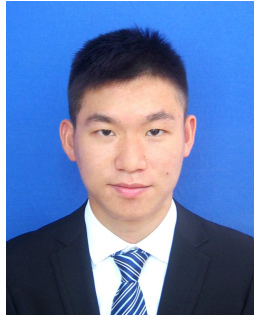

Shu-Lin Chen (S'16) was born in Hubei Province, China. He received the B.S. degree in electrical engineering from Fuzhou University, China, in 2012, and the M.S. degree in electromagnetic field and microwave technology from Xiamen University, China, in 2015. Since 2016, he has been working towards the $\mathrm{PhD}$ degree in Engineering with the Global Big Data Technologies Centre (GBDTC), University of Technology Sydney (UTS), Australia.

His research interests include reconfigurable antennas, leaky-wave antennas, millimeter wave antennas, and adaptive array processing. He was a finalist of ISAP 2017 best paper competition, and his paper was listed as an Honorary Mention in APS-URSI 2017.

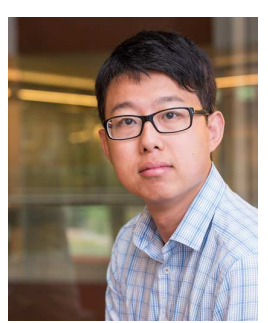

Zheng Li (M'15) received the B.S. degree in physics and $\mathrm{Ph} . \mathrm{D}$. degree in electrical engineering from Beijing Jiaotong University, Beijing, China, in 2006 and 2012, respectively. From 2008 to 2009, he was a visiting student with the Pennsylvania State University, Pennsylvania, USA. From 2017 to 2018 , he was a visiting professor with the Global Big Data Technologies Centre, University of Technology Sydney, Australia.

In 2012, he joined the faculty of the Department of Electrical Engineering, Beijing Jiaotong University, where he became an associate professor in 2015. His research interests include leaky-wave antennas and leaky waveguides.

Pei-Yuan Qin (M'13) was born in Liaoning Province, China, in 1983. He received a Bachelor Degree in Electronic Engineering from Xidian University, Xi'an, China, in 2006, and a joint Ph.D. Degree from Xidian University and Macquarie University, Australia, in electromagnetic fields and microwave technology in 2012.

From 2012 to 2015, he was a Postdoctoral Research Fellow in Commonwealth Scientific and Industrial Research Organisation (CSIRO), Australia. From 2015 to 2016, he was a Chancellor's Postdoctoral Research Fellow/Lecturer with University of Technology Sydney (UTS), Australia. Since 2017, he is a Senior Lecturer with UTS. His research interests are in the areas of reconfigurable antennas, phase shifters, reconfigurable reflectarrays, and MIMO communications.

Dr. Qin was a recipient of an Australia Research Council Discovery Early Career Researcher Award and also a recipient of the International Macquarie University Research Excellence Scholarship. He was awarded the Vice Chancellor's Commendation for academic excellence by Macquarie University. Since 2017, he has been serving as an Associate Editor of the IEEE Antennas and Wireless Propagation Letters. 


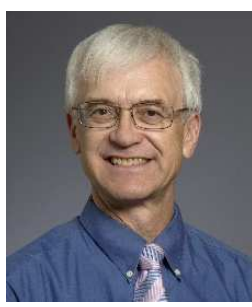

Richard W. Ziolkowski (M'87-SM'91-F'94) received the B.Sc. (magna cum laude) degree (Hons.) in physics from Brown University, Providence, RI, USA, in 1974; the M.S. and Ph.D. degrees in physics from the University of Illinois at UrbanaChampaign, Urbana, IL, USA, in 1975 and 1980, respectively; and the Honorary Doctorate degree from the Technical University of Denmark, Kongens Lyngby, Denmark in 2012.

$\mathrm{He}$ is currently a Distinguished Professor in the Global Big Data Technologies Centre in the Faculty of Engineering and Information Technologies (FEIT) at the University of Technology Sydney, Ultimo NSW, Australia. He became a Professor Emeritus at the University of Arizona in 2018, where he was a Litton Industries John M. Leonis Distinguished Professor in the Department of Electrical and Computer Engineering in the College of Engineering and was also a Professor in the College of Optical Sciences. He was the Computational Electronics and Electromagnetics Thrust Area Leader with the Lawrence Livermore National Laboratory, Engineering Research Division, in Livermore, CA before joining The University of Arizona, Tucson, AZ, USA, in 1990.

Prof. Ziolkowski is the recipient of the 2019 IEEE Electromagnetics Award (IEEE Field Award). He is a Fellow of the Optical Society of America (OSA, 2006), and of the American Physical Society (APS, 2016). He was the Australian DSTO Fulbright Distinguished Chair in Advanced Science and Technology from 2014-2015. He was a 2014 Thomas-Reuters Highly Cited Researcher. He served as the President of the IEEE Antennas and Propagation Society in 2005 . He is also actively involved with the URSI, OSA and SPIE professional societies.

His current research interests include the application of new mathematical and numerical methods to linear and nonlinear problems dealing with the interaction of electromagnetic and acoustic waves with complex linear and nonlinear media, as well as metamaterials, metamaterial-inspired structures, nano-structures, and other classical and quantum applications-specific configurations.

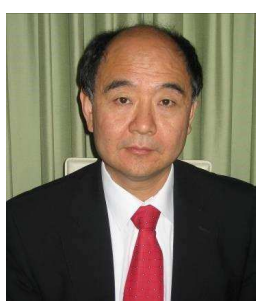

Y. Jay Guo (Fellow'2014) received a Bachelor Degree and a Master Degree from Xidian University in 1982 and 1984, respectively, and a PhD Degree from Xian Jiaotong University in 1987, all in China. His research interest includes antennas, mm-wave and $\mathrm{THz}$ communications and sensing systems as well as big data technologies. He has published over 400 research papers and holds 24 patents in antennas and wireless systems. He is a Fellow of the Australian Academy of Engineering and Technology, a Fellow of IEEE and a Fellow of IET, and a member of the College of Experts of Australian Research Council (ARC). He has won a number of most prestigious Australian national awards, and was named one of the most influential engineers in Australia in 2014 and 2015.

Prof. Guo is a Distinguished Professor and the founding Director of Global Big Data Technologies Centre at the University of Technology Sydney (UTS), Australia. Prior to this appointment in 2014, he served as a Director in CSIRO for over nine years, directing a number of ICT research portfolios. Before joining CSIRO, he held various senior technology leadership positions in Fujitsu, Siemens and NEC in the U.K.

Prof. Guo has chaired numerous international conferences. He is the Chair Elect of International Steering Committee, International Symposium on Antennas and Propagation (ISAP). He was the International Advisory Committee Chair of IEEE VTC2017, General Chair of ISAP2015, iWAT2014 and WPMC'2014, and TPC Chair of 2010 IEEE WCNC, and 2012 and 2007 IEEE ISCIT. He served as Guest Editor of special issues on "Antennas for Satellite Communications" and "Antennas and Propagation Aspects of 6090GHz Wireless Communications," both in IEEE Transactions on Antennas and Propagation, Special Issue on "Communications Challenges and Dynamics for Unmanned Autonomous Vehicles," IEEE Journal on Selected Areas in Communications (JSAC), and Special Issue on "5G for Mission Critical Machine Communications", IEEE Network Magazine. 